\title{
Desempenho funcional de crianças com deficiência visual, atendidas no Departamento de Estimulação Visual da Fundação Altino Ventura
}

\author{
Functional outcome of visually handicapped children cared for at the \\ Departmentof Visual Stimulation - "Fundação Altino Ventura"
}

\author{
Juliana Malta1 \\ Daniela Endriss ${ }^{2}$ \\ Sueli Rached ${ }^{3}$ \\ Terezinha Moura ${ }^{4}$ \\ Liana Ventura ${ }^{5}$
}

\begin{tabular}{|l|}
\hline RESUMO \\
\hline Objetivo: Traçar o perfil funcional de crianças portadoras de deficiência \\
visual. Métodos: Foram avaliadas 27 crianças. Dezessete com acuidade \\
visual normal (que freqüentavam a Escola Municipal Alto do Maracanã, \\
em Recife, e dez portadoras de deficiência visual (que recebiam estimulação \\
visual no Departamento de Estimulação Visual da Fundação Altino \\
Ventura, Recife). Foi utilizado o teste funcional padrão PEDI. Resultados: \\
As crianças portadoras de deficiência visual apresentaram desempenho \\
significantemente inferior nas áreas de autocuidado e mobilidade que as \\
crianças do grupo controle. Conclusão: A deficiência visual traz conse- \\
qüências limitantes para o desempenho de atividades de autocuidado, \\
locomoção, compreensão, comunicação e tarefas domésticas, na faixa \\
etária estudada.
\end{tabular}

Descritores: Portadores de deficiência visual; Acuidade visual; Criança; Autocuidado; Bem estar da criança
${ }^{1}$ Residente do $1^{\circ}$ ano da Fundação Altino Ventura Recife (PE) - Brasil.

${ }^{2}$ Médica oftalmologista da Fundação Altino Ventura e Hospital de Olhos de Pernambuco - Recife (PE) - Brasil.

${ }^{3}$ Pedagoga do Departamento de Estimulação Visual da Fundação Altino Ventura e Hospital de Olhos de Pernambuco - Recife (PE) - Brasil.

${ }^{4}$ Terapêutica ocupacional e Pedagoga da Escola Especial Instituto de Cegos - Recife (PE) - Brasil.

5 Doutora em Medicina pela UFMG; Coordenadora do Curso de Residência Médica, Especialização e Fellow da Fundação Altino Ventura - Recife (PE) - Brasil.

Endereço para correspondência: Fundação Altino Ventura. Rua da Soledade, 170 - Boa Vista - Recife (PE) CEP 50070-040

E-mail: fav@fundacaoaltinoventura.org.br

Recebido para publicação em 29.06.2005

Versão revisada recebida em 20.01.2006

Aprovação em 16.02.2006

\section{INTRODUÇÃO}

As causas de cegueira na infância variam de região para região, sendo, em parte, determinada pelo desenvolvimento socioeconômico, e a disponibilidade de serviços de saúde. Dentre as 1,5 milhões de crianças cegas no mundo $75 \%$ apresentam causas preveníveis ou curáveis ${ }^{(1-3)}$. Se a criança se torna deficiente visual após os cinco anos de idade, ela já terá desenvolvido praticamente todo o seu potencial visual, poderá conservar as imagens e memória visual. As crianças que nascem cegas ou perderam a visão muito cedo, apresentam necessidades de aprendizagem diferentes das demais crianças $^{(4)}$.

A aprendizagem por meio de um sistema visual alterado, embora se produza mais lentamente e inclusive em alguns casos de forma adaptada, segue o mesmo processo de desenvolvimento que um sistema visual normal, sendo fundamental nesse momento a aplicação de programas voltados a promover a eficiência visual. A potencialidade de cada criança para aprender a interagir em condições satisfatórias em seu meio familiar, na escola e em seu ambiente social pode ser estimulada ou inibida pela atitude das pessoas que a rodeiam ${ }^{(5)}$.

A deficiência visual, visão pior que 20/60, impõe restrições à capacidade de movimento livre, seguro e confiante da criança no ambiente. Agir autonomamente é fundamental para uma criança com deficiência visual, pois, o 
movimento é fonte de ação, da experiência, da integração sensorial, da aprendizagem e construção do conhecimento ${ }^{(4)}$. Mais recentemente, novas abordagens de avaliação têm sido desenvolvidas com base nestas tendências teóricas. Estas avaliações apresentam-se como testes padronizados que documentam de forma objetiva e quantitativa características funcionais da criança, possibilitando assim a investigação sistemática de aspectos como a qualidade do movimento e a mobilidade $^{(6-7)}$.

O teste funcional "Pediatric Evalution of Disability Inventory" (PEDI), é uma avaliação padronizada do desempenho da criança em atividades e tarefas típicas da rotina doméstica. Trabalhos anteriores foram realizados utilizando o PEDI para avaliar o desempenho funcional de crianças deficientes ${ }^{(6,8-10)}$.

O presente estudo tem por objetivo descrever o padrão de desenvolvimento das habilidades funcionais de autocuidado em crianças com deficiência visual, observando as dificuldades relativas no desempenho de atividades funcionais de alimentação, banho, vestir e higiene pessoal, utilizando o método de avaliação PEDI.

\section{MÉTODOS}

O estudo foi realizado de agosto a novembro de 2004, na Escola Municipal Alto do Maracanã - Recife e no Departamento de Estimulação Visual da Fundação Altino Ventura. A investigação foi prospectiva do tipo corte transversal.

O grupo sem deficiência visual (controle) foi constituído por 17 crianças, sendo oito $(47,0 \%)$ do sexo masculino e nove $(53,0 \%)$ do feminino. A idade variou de 70 a 90 meses, com média de $82 \pm 6$ meses. Os pais das crianças foram entrevistados na escola da rede municipal de ensino da cidade do Recife.

O grupo de deficientes visuais foi constituído por 10 crianças, sendo seis $(60,0 \%)$ do gênero masculino e quatro $(40,0 \%)$ do feminino. Neste grupo, a idade variou de 58 a 96 meses (média de $80 \pm 12$ meses). Os pais das crianças foram entrevistados durante as consultas no Departamento de Visão Subnormal no período de agosto a novembro de 2004. As crianças desse grupo apresentavam, no melhor olho, acuidade visual corrigida pior que 20/60. Foram excluídas deste estudo crianças que apresentavam distúrbios associados, como retardo mental, epilepsia, déficit auditivo e distúrbios de linguagem.

As crianças foram avaliadas pelo teste PEDI, que foi traduzido para o português e adaptado para contemplar as especificidades socioculturais do Brasil, com permissão e colaboração dos autores da avaliação original ${ }^{(7)}$. O PEDI é uma avaliação realizada através de entrevista com pais ou responsáveis que possam informar sobre o desempenho da criança em casa. Este teste avalia aspectos funcionais do desempenho de crianças com idade entre 6 meses e 7 anos e meio, em três áreas de desempenho: autocuidado, mobilidade, função social. Este teste pode ser usado em crianças com idades cronológicas superiores ao limite indicado, desde que o desempenho funcional das mesmas esteja aquém desse limite.
A primeira parte do teste avalia as habilidades ou capacidades funcionais da criança; a segunda parte informa sobre a quantidade de ajuda ou assistência que a criança recebe para desempenhar as atividades funcionais, e a terceira parte documenta as modificações do ambiente necessárias para o desempenho de tarefas funcionais.

O desempenho funcional foi avaliado em três aspectos do desenvolvimento: autocuidado (73 itens), mobilidade (59 itens) e função social (65 itens). Cada item é pontuado com escore 0 (zero) se a criança não é capaz de desempenhar a atividade funcional, ou 1 ( $\mathrm{um}$ ) se a atividade fizer parte do repertório de atividades da criança. Os 73 itens funcionais avaliados na escala de autocuidado foram agrupados nas seguintes tarefas: alimentação (14 itens), higiene pessoal (14 itens), banho (10 itens), vestir (20 itens), uso do toalete (5 itens) e controle esfincteriano (10 itens). Os 59 itens funcionais da escala de mobilidade são agrupados nas seguintes tarefas: transferências (24 itens), locomoção em ambientes internos (13 itens), locomoção em ambientes externos (12 itens) e uso da escada (10 itens). Na escala de função social, os 65 itens ou habilidades funcionais foram agrupados da seguinte forma: compreensão funcional (15 itens), verbalização (10 itens), resolução de problemas (5 itens), brincar (15 itens), autoinformação (5 itens), participação da rotina doméstica/comunidade (10 itens) e noção de autoproteção (5 itens).

A pontuação da segunda parte do PEDI é dada em uma escala ordinal que varia de 5 (se a criança é independente no desempenho da tarefa, não necessitando de nenhum tipo de assistência do cuidador para realizar a tarefa) a 0 (se a criança é totalmente dependente do cuidador para realizar a tarefa). Os escores intermediários descrevem quantidades variadas de ajuda fornecida pelo cuidador (supervisão: mínima, moderada ou máxima).

Neste estudo foram utilizadas apenas as três escalas das atividades funcionais da parte I do teste PEDI.

As crianças foram avaliadas por uma terapeuta ocupacional previamente treinada na aplicação do teste. Cada entrevista durou cerca de 45 minutos.

Os dados deste estudo foram analisados através do teste $\mathrm{t}$ de Student para grupos independentes. Foram comparadas as médias dos escores obtidos pelas crianças dos diferentes grupos, nas áreas do desempenho funcional (autocuidado, mobilidade e função social). Para cada análise foi considerado nível de significância de $95 \%(\mathrm{p}<0,05)$.

O projeto de investigação foi aprovado pelo Comitê de Ética da Fundação Altino Ventura. O responsável por cada criança foi previamente informado sobre os objetivos e procedimentos deste estudo e convidado a assinar um termo de consentimento livre autorizando a participação de seu (sua) filho(a).

\section{RESULTADOS}

Na tabela 1 observam-se os resultados das comparações entre as médias dos escores dos dois grupos, nas três áreas de desempenho funcional. 


\begin{tabular}{|c|c|c|c|c|c|c|}
\hline \multirow[b]{2}{*}{ Área } & \multicolumn{6}{|c|}{ Medidas descritivas } \\
\hline & Grupo & Valor mínimo & Mediana & Valor máximo & Média \pm DP & $p$ \\
\hline \multirow[t]{2}{*}{ Autocuidado } & Controle & 66 & 71 & 73 & $70 \pm 2,2$ & $p<0,01$ \\
\hline & DV & 53 & 64 & 71 & $63 \pm 7,3$ & \\
\hline \multirow[t]{2}{*}{ Mobilidade } & Controle & 54 & 59 & 62 & $59 \pm 1,8$ & $p<0,01$ \\
\hline & DV & 42 & 58 & 59 & $54 \pm 6,2$ & \\
\hline \multirow[t]{2}{*}{ Função social } & Controle & 42 & 59 & 64 & $58 \pm 5,3$ & $p=0,06$ \\
\hline & DV & 36 & 54 & 63 & $53 \pm 7,2$ & \\
\hline
\end{tabular}

As crianças portadoras de deficiência visual apresentaram desempenho significantemente inferior ao das crianças do grupo controle na habilidade funcional de autocuidado $(\mathrm{p}<0,01)$ e mobilidade $(\mathrm{p}<0,01)$. A média dos escores da função social foi maior no grupo controle porém não alcançou significância estatística $(\mathrm{p}=0,06)$.

\section{DISCUSSÃO}

A deficiência visual impõe restrições ao desenvolvimento seguro e confiante da criança no ambiente, interfere no conhecimento do próprio corpo, e na inter-relação entre as coisas e as pessoas no ambiente ${ }^{(4)}$. A promoção do desempenho de atividades e tarefas funcionais pode ser definida como objetivo a ser alcançado pelas terapêuticas empregadas. No presente estudo foi observada a influência da deficiência visual no desempenho funcional das crianças, principalmente em atividades de autocuidado e mobilidade, em que houve uma diferença estatisticamente significante comparando-se crianças sem deficiências e deficientes visuais.

Os sentimentos de pena, piedade e comiseração são freqüentes em relação à criança deficiente visual, levando muitas vezes a atitudes de superproteção, como protegê-la em casa, guiar ou conduzi-la, evitar que brinque com crianças de visão normal, deixá-la sentada para que não se machuque, falar por ela ou tentar resolver os seus problemas ${ }^{(4)}$. Esta atitude pode interferir na independência dessas crianças para desempenhar atividades. Em vista disso, durante a estimulação visual é importante enfatizar a necessidade da participação dessas crianças nas atividades diárias. A aceitação da deficiência visual significa a aceitação da pessoa como ela é, sem o desejo de transformá-la ou modificá-la para que seja aquilo que se considera o melhor ${ }^{(11)}$.

A interação social depende da forma de como a criança é recebida, acolhida, observada, ouvida e compreendida em suas necessidades. Essa forma de relação e comunicação influencia o desenvolvimento psicoafetivo e determina a maneira como a criança vai interagir com as pessoas, objetos e o meio em que vive ${ }^{(4)}$. Quanto à função social, foi observado no presente estudo, a média dos escores maior nas crianças do grupo controle, no entanto, esta diferença não foi estatisticamente significante, possivelmente devido ao tamanho reduzido da amostra.
A comparação entre os escores nas diferentes faixas etárias pode mostrar o momento onde as dificuldades nas áreas testadas são maiores e em que faixa etária essas diferenças entre os escores são menores. Estudos realizados com crianças portadoras de síndrome de Down com 2 e 5 anos indicam que as diferenças observadas entre os dois grupos são influenciadas pela idade, não permanecendo constante ao longo do desenvolvimento ${ }^{(9)}$. Com esses dados sabe-se quando e onde intervir para o melhor desempenho e reinserção social.

Apesar do pioneirismo na avaliação objetiva do desempenho funcional de crianças deficientes utilizando o PEDI, este foi um estudo transversal avaliando as crianças em apenas um momento. Idealmente, o PEDI deve ser aplicado a cada seis meses para possibilitar a identificação das áreas deficientes como também o acompanhamento e avaliação das intervenções realizadas.

\section{CONCLUSÃO}

No estudo observam-se as limitações significantes que crianças deficientes visuais apresentam no desempenho funcional da rotina diária. Essas limitações manifestaram-se principalmente nas áreas de autocuidado e mobilidade. Estes resultados podem ser utilizados para o melhor planejamento das atividades de reabilitação visual e integração social destas crianças. Para esta integração, essas crianças além da estimulação visual necessitam também de um time integrado de outros profissionais como: terapeuta ocupacional, psicólogos, assistente social e pedagogos.

Estudos futuros, com amostras pareadas, analisando-se as crianças antes e após o início da estimulação visual, em diversas faixas etárias podem ser úteis no conhecimento sobre o impacto desta atividade no perfil funcional das crianças.

\section{ABSTRACT}

Purpose: To analyze the functional outcome of visually handicapped children. Methods: Twenty-seven children were evaluated using the standard functional PEDI test. Seventeen who attended school at "Escola Municipal Alto do Maracanã", Recife - Pernambuco, Brazil, had normal visual acuity and the other ten had visual handicap, and were cared for at 
574 Desempenho funcional de crianças com deficiência visual, atendidas no Departamento de Estimulação Visual da Fundação Altino Ventura

Stimulation Visual Department - "Fundação Altino Ventura", in the same city. Results: The children who had visual handicap showed significantly worse performance in self-care and mobility than normal children. Conclusion: Visual handicap may have limited the performance regarding self-care, mobility, understanding, communication, home works, in the studied group.

Keywords: Visually impaired persons; Visual acuity; Child; Self-care; Child welfare

\section{REFERÊNCIAS}

1. Wilson ME, Pandey SK, Thakur J. Paediatric cataract blindness in the developing world: surgical techniques and intraocular lenses in the new millenium. $\mathrm{Br}$ J Ophthalmol. 2003;87(1):14-9. Review.

2. Gilbert C, Foster A. Childhood blindness in the context of VISION 2020 - the right to sight. Bull World Health Organ. 2001;79(3):227-32. Epub 2003 Jul 7.

3. Hoyt CS, Good WV. The many challenges of childhood blindness. Br J Ophthalmol. 2001;85(10):1145-6.

4. Brasil. Ministério da Educação. Saberes e práticas da inclusão: dificuldades de comunicação e sinalização. Deficiência visual. Educação Infantil vol. 08. Brasília: MEC/SEESP; 2004.

5. Martín MB, Bueno ST. Deficiência visual: aspectos psicoevolutivos e educativos. São Paulo: Editora Santos; 2003.

6. Mancini MC, Teixeira S, Araújo LG, Paixão ML, Magalhães LC, Coelho ZAC, et al. Estudo do desenvolvimento da função motora aos 8 e 12 meses de idade em crianças nascidas pré-termo e a termo. Arq Neuropsiquiatr. 2002;60 (4):974-80.

7. Haley SM, Coster WJ, Ludlow LH, Haltiwanger JT, Andrelow PJ. Inventário de avaliação pediátrica de disfunção: versão brasileira. Tradução e adaptação cultural: Mancini MC. Belo Horizonte: Laboratórios de Atividade e Desenvolvimento Infantil. Departamento de Terapia Ocupacional. Universidade Federal de Minas Gerais; 2000.

8. Allegretti ALC, Mancini MC, Schwartzman JS. Estudo do desempenho funcional de crianças com paralisia cerebral diparética espástica utilizando o Pediatric Evalution of Disability Inventory (PEDI). Temas Desenvolv. 2002;11(64):5-11.

9. Mancini MC, Fiuza PM, Rebelo JM, Magalhães LC, Coelho ZAC, Paixão ML, et al. Comparação do desempenho de atividades funcionais em crianças com desenvolvimento normal e crianças com paralisia cerebral. Arq Neuropsiquiatr. 2002;60(2B):446-52.

10. Mancini MC, Silva PC, Gonçalves SB, Martins SM. Comparação do desempenho funcional de crianças portadoras de síndrome de Down e crianças com desenvolvimento normal aos 2 e 5 anos de idade. Arq Neuropsiquiatr. 2003;61 (2B):409-15.

11. Amiralian MLTM. O psicólogo e a pessoa com deficiência visual. In: Masini EFS, organizador. Do sentido... pelos sentidos... para o sentido. São Paulo: Vetor Editora; 2002; p.201.

\section{Stmposio do Cornea e Claucoma
do Hospital Citalmologico de Sorocaba}

\section{0 - 21 de Outubro de 2006 Sorocaba - SP}

INFORMAÇÕES: Tel.: (15) 3212-7077

E-mail: ceo@hosbos.com.br 